\title{
On-demand Influencer Discovery on Social Media
}

\author{
Cheng Zheng ${ }^{1}$, Qin Zhang $^{2}$, Sean D. Young ${ }^{3}$, and Wei Wang ${ }^{1}$ \\ ${ }^{1}$ Department of Computer Science, University of California, Los Angeles \\ ${ }^{2}$ Center for Artificial Intelligence, FEIT, University of Technology Sydney \\ ${ }^{3}$ Departments of Emergency Medicine and Informatics, University of California, Irvine \\ \{chengzheng,weiwang\}@cs.ucla.edu,Qin.Zhang@student.uts.edu.au,syoung5@uci.edu
}

\begin{abstract}
Identifying influencers on social media, such as Twitter, has played a central role in many applications, including online marketing and political campaigns. Compared with social media celebrities, domain-specific influencers are less expensive to hire and more engaged in spreading messages such as new treatment or timely prevention for HIV. However, most of the existing topic modeling based approaches fail to identify influencers who are dedicated to the rare yet important topics such as HIV and suicide. To alleviate this limitation, we investigate an on-Demand Influencer Discovery (DID) framework that is able to identify influencers on any subject depicted by a few user-specified keywords, regardless of its popularity on social media. The DID model employs an iterative learning process that integrates the language attention network as a subject filter and the influence convolution network built on user interactions. Comprehensive evaluations on Twitter datasets show that the DID model can reliably identify influencers even on rare subjects such as HIV and suicide, outperforming existing topic-specific influencer detection models.
\end{abstract}

\section{KEYWORDS}

Topic-specific Influencers, Rare Topics, Influence Convolution

\section{ACM Reference Format:}

Cheng Zheng ${ }^{1}$, Qin Zhang ${ }^{2}$, Sean D. Young ${ }^{3}$, and Wei Wang ${ }^{1}$. 2020. Ondemand Influencer Discovery on Social Media. In Proceedings of the 29th ACM International Conference on Information and Knowledge Management (CIKM '20), October 19-23, 2020, Virtual Event, Ireland. ACM, New York, NY, USA, 4 pages. https://doi.org/10.1145/3340531.3412134

\section{INTRODUCTION}

Social influence refers to the ability of a user to change the feelings, attitudes, or behaviors of other users within a network [4]. Detecting influencers has become an essential task in many fields such as online marketing and political campaigns [15, 20]. Many previous works have studied the task by utilizing the rich text and user interactions on social media $[2,17,19]$.

It is well accepted that the influence distribution is not homogeneous over topics. An influencer on topics about politics may not have a huge impact on topics about HIV. Compared with social media celebrities, domain-specific influencers are less expensive to

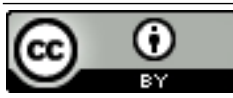

This work is licensed under a Creative Commons Attribution International 4.0 License. CIKM '20, October 19-23, 2020, Virtual Event, Ireland

(C) 2020 Copyright held by the owner/author(s).

ACM ISBN 978-1-4503-6859-9/20/10.

https://doi.org/10.1145/3340531.3412134

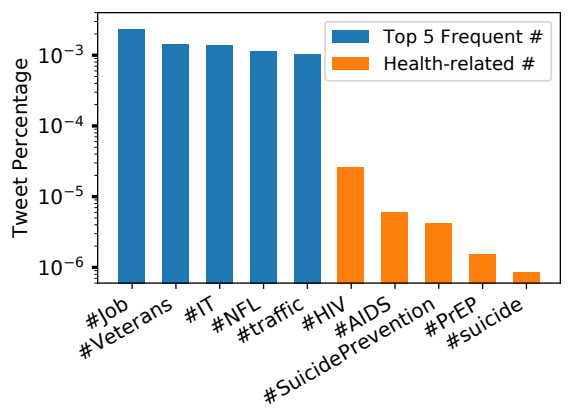

Figure 1: Hashtag distribution of 1\% US English Tweets in 11/01/2019-12/31/2019. The top 5 frequent hashtags are shown along with 5 health-related hashtags.

hire and more engaged in spreading messages such as new treatment or timely prevention for HIV. However, these important topics are rarely discussed on social media, which is demonstrated with an example from Twitter in Figure 1. We crawled 1\% US English Tweets during 11/01/2019 - 12/31/2019 and counted the occurrence of hashtags, with the top 5 frequent hashtags in blue and 5 rare but important hashtags on health subjects in orange. Although topic-specific influencer detection algorithms [1, 2, 7, 15] have been proposed, none of them focuses on identifying influencers for rare yet important topics like HIV and suicide. While existing approaches trying to build filters with keywords [6], it is infeasible to enumerate all keywords related to the topic (like $\# P r E P^{1}$ ).

Another key question in identifying topic-specific influencers is how to model the influence propagation. Traditional influencer detection models rely on topic modeling $[7,15]$ and probabilistic network diffusion $[8,17]$, which are not ideal for the sparse social network with rare topics. Meanwhile, the recent progress on Graph Neural Networks (GNNs) $[9,16,18]$ demonstrates the effective representation learning in graph structures. With a proper design of neighborhood aggregation and objective function, GNNs are capable of modeling the influence propagation and identifying topic-specific influencers on social media.

To tackle the challenges of detecting on-demand topic-specific influencers, we propose a new computational framework named onDemand Influencer Discovery (DID) model. We design an language attention network as a subject filter to iteratively select the social messages related to given keywords. To take full advantage of multiple interactions in social networks, we integrate them with trainable weight functions. The influence propagation is modeled by GNNs with a loss function considering neighborhood and topic

\footnotetext{
${ }^{1}$ Pre-exposure prophylaxis (PrEP) is a prevention of HIV/AIDS.
} 


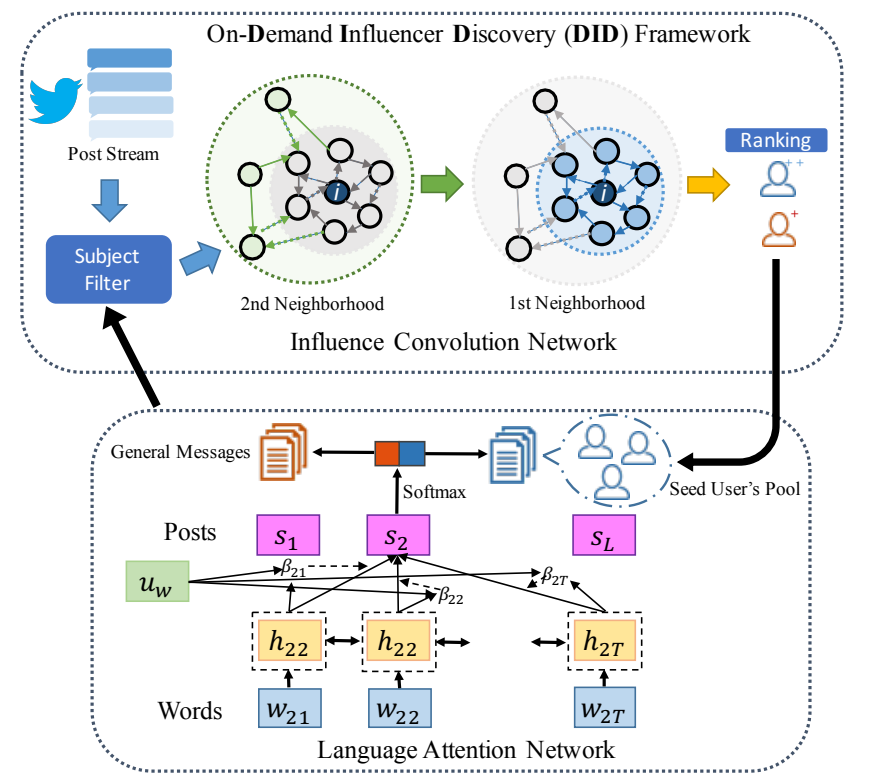

Figure 2: The overview of the proposed framework, DID

concentration. Comprehensive experiments show our approach significantly outperforms all comparative baselines.

In the literature, influencer detection has attracted increasing attention in recent years. Some of the existing methods focus on modeling the influence in all topics $[3,13,17]$, while several studies aim to identify topic-specific influencers [12, 14, 15]. Although some approaches $[1,2,7]$ also integrate topic discovery and social influence analysis, the influencers are restricted to popular topics in social media only. To the best of our knowledge, this work is the first study that can reliably identify topic-specific influencers even on rare but important subjects.

\section{ON-DEMAND INFLUENCER DISCOVERY}

In this section, we formally define the problem statement and then introduce our proposed approach, on-Demand Influencer Discovery (DID).

Problem Definition. Suppose we have a set of social media users $U$ and their social network $G$. For each user $k \in U, W^{k}=\left\{w_{i j}^{k}\right\}$ denotes the social media posts published by the user, where $w_{i j}^{k}$ represents the $j$-th word of the $i$-th post in $W^{k}$. The social network $G=(U, A)$ treats each user $k \in U$ as a node and models their relations with the adjacency matrix $A \in \mathbb{R}^{N \times N}$ that indicates the relations between users. $N$ is the total number of users $(|U|=N)$. Given the social media posts of users $U$ and the social network $G$, the goal of this work is to identify top- $t$ influencers with the given keyword set $K$.

Framework Overview. Here we propose DID for the on-demand influencer detection as shown in Figure 2. We design the language attention network that can select subject-related social posts and get trained iteratively with minimum supervision. The influence convolution network simulates the influence propagation in the social network and outputs the topic-specific influence scores of users. Moreover, we integrate different types of social interactions with weight functions that are trained with influence learning.

\subsection{Language Attention Network}

We propose the language attention network as a subject filter to select social posts related to the given keyword set $K$. The language attention network is composed of several parts, including a word embedding layer, a post encoder, and a subject classifier.

Word Embedding Layer. We convert each word $w_{i j}$ into a onehot encoding representation $\tilde{w}_{i j}$ and embed the words to vectors $e$ with an embedding matrix $E$, where $e_{i j}=E \cdot \tilde{w}_{i j}$.

Post Encoder. For each post of the user, we feed the word embeddings to a bidirectional Recurrent Neural Network (BiRNN) to learn a hidden state of each word with sequential information as:

$\overleftarrow{h}_{i j}=\operatorname{GRU}\left(\overleftarrow{h}_{i, j+1}, e_{i j}\right), \vec{h}_{i j}=\operatorname{GRU}\left(\vec{h}_{i, j-1}, e_{i j}\right), h_{i j}=\left[\overleftarrow{h}_{i j}, \vec{h}_{i j}\right]$

where GRU(.) is the recurrent neural unit of the BiRNN. Here we choose GRU instead of LSTM because of its computational efficiency. To derive the post representation, we introduce an attention layer to obtain a weighted sum of the hidden states from the BiRNN layer. To be specific, we initialize a context vector $u_{w}$ and calculate the attention scores $\beta_{i j}$ for the words in the post as:

$u_{i j}=\tanh \left(W_{w} \cdot h_{i j}+b_{w}\right), \beta_{i j}=\frac{\exp \left(u_{i j}^{T} \cdot u_{w}\right)}{\sum_{j} \exp \left(u_{i j}^{T} \cdot u_{w}\right)}, s_{i}=\sum_{j} \beta_{i j} \cdot h_{i j}$,

where $W_{w}$ and $b_{w}$ are the weight matrix and the bias to map each word into a hidden space for estimating importance. With the post encoder, the $i$-th social post is represented as $s_{i}$.

Subject Classifier. As a subject filter, the language attention network aims to select social posts related to the given keywords. We build a subject seed user pool to capture as much subject-related information in the social posts. Subject seed users are defined as users who are dedicated to the topic of given keywords. We initialize the pool by selecting the users that have the highest ratio of posts containing keyword set $K$. Furthermore, as shown by the right black arrow in Figure 2, we iteratively update the seed user pool and subject classifier. More seed users are incorporated into the pool according to the rules: (1) the user has a high ratio of subject-related posts; (2) the user is identified as an influencer. For example in HIV subject on Twitter, the seed user pool is initialized with @iasociety and @CDC_HIVAIDS, who have the highest 73.4\% and 79.5\% of tweets containing keyword $H I V$. As the training continues, we have 6 users in the final seed user pool. By collecting posts from seed users as positive samples and general posts as negative samples, we train the subject classifier and iteratively update the seed user pool. Based on the post encoding $s_{i}$, we use a fully-connected hidden layer to estimate the probability of being the positive sample of the $i$-th social post as

$$
P=\operatorname{Softmax}\left(W_{c} s_{i}\right),
$$

where $W_{c}$ is the weight matrix for the subject classifier. With the subject classifier, we obtain the subject-related posts and a vector $\mathbf{c} \in \mathbb{R}^{N}$ with $\mathbf{c}_{i}=\frac{\text { \# related posts of } i}{\text { \# total posts of } i}$, describing user $i$ 's concentration on topic $K$. Consequently, the filtered social network only includes interactions from the subject-related posts. 


\subsection{Influence Convolution Network}

Interaction Integration. Typically, the social network includes multiple types of interactions, such as follow, mention, retweet and quote on Twitter. We assume there are $L$ types of interactional graph and the corresponding adjacency matrices are $A^{(1)}, A^{(2)}, \ldots, A^{(L)}$ The majority of existing works only consider a single graph or assign equal weights to different interaction graphs [5]. Instead, we would like to integrate the multiple graphs in a sensible way by learning the weights of the integration. Specifically, we introduce $L$ coefficients $\left\{\lambda_{l}, l=1,2, \ldots, L\right\}$ to represent the weights for $L$ adjacency matrices $A^{(l)}$. The integrated adjacency matrix can be formulated as $A=\sum_{l=1}^{L} \lambda_{l} A^{(l)}$, with $\lambda_{l}$ to be learned.

Influence Convolution. On social media, users disseminate posts via multiple rounds of social actions. Based on the closed world assumption [11], social actions are mostly influenced by their near neighbors within the network. As a result, we propose to model the influence propagation on social media with neighborhood aggregation technique from GNNs [9], as shown in Figure 2. The post embedding $s$ aggregated by user is the input node representations $H^{(0)}$ to the first layer. The $p$-th influence convolution layer performs feature aggregation on node $i$ 's one-hop neighbors $\mathbb{N}_{i}$ :

$$
H^{(p)}=\sigma\left(\sum_{j \in \mathbb{N}_{i}} H_{j}^{(p-1)} W^{(p)}\right)
$$

where $H^{(p)} \in \mathbb{R}^{N \times d_{n}^{(p)}}$ is the output node representations, and $W^{(p)} \in \mathbb{R}^{d_{n}^{(p-1)} \times d_{n}^{(p)}}$ is the parameter matrix for this layer. The aggregated feature matrix $H$ from the output of the final layer represents the user influence after propagation. We consider three criteria for the unsupervised objective function. First, users with a larger neighborhood should have a higher influence score. Here we consider the neighborhood within two hops. Second, the higher the concentration $\mathbf{c}$ in keyword topic $K$, the higher the influence score. Furthermore, we add the third term in the objective function to regularize the weight matrices of $P$ influence convolution layers to prevent overfitting of the model.

$$
\begin{aligned}
\max L\left(W, \lambda_{l}\right) & =\sum_{i, j=1}^{N} A_{i j}\left(1+\sum_{k=1}^{N} A_{j k}\right) H_{i}^{2} \\
& +\zeta_{1} \mathbf{c}^{T} H-\zeta_{2} \sum_{i=1}^{P}\left\|W^{(i)}\right\|^{2}
\end{aligned}
$$

\section{EXPERIMENTS}

\subsection{Experimental Setup}

Datasets. We employ three datasets from Twitter. For the quantitative assessment, we create HIV and SuICIDE datasets. For HIV dataset, we select a sample of users who tweeted about HIV and crawl all their tweets. We label the HIV-related influencers as the users (1) who have more than 50\% HIV-related tweets and (2) whose HIV-related tweets have the highest number of retweets in the whole Twitter network. We adopt the same strategy to create SuICIDE dataset focusing on the suicide-related topic. There are 20 and 15 topic-specific influencers in the two labeled datasets. The US-ENGLISH includes randomly sampled 1\% US tweets in 11/01/2019 $-12 / 31 / 2019$ and the hashtag distribution is shown in Figure 1. We intend to evaluate the scalability and efficiency with the unlabeled US-ENGLISH dataset. Dataset statistics are summarized in Table 1.

Table 1: Dataset statistics.

\begin{tabular}{cccc}
\hline & HIV & SUICIDE & US-ENGLISH \\
\hline Users & 556 & 639 & $4,145,234$ \\
Tweets & 341,167 & 471,625 & $75,546,211$ \\
Interactions & 81,626 & 89,776 & $3,848,428$ \\
Influencers & 20 & 15 & - \\
\hline
\end{tabular}

Baselines. We compare the proposed DID against six baselines that are trained based on the text and network features. The first three baselines are topic-specific influencer detection models: (1) TAP [12] a topical affinity propagation model built on a factor graph to identify the topic-specific social influence; (2) TwitterRank [15], an extension of PageRank algorithm with topic modeling; (3) TSSRW [7], a topic-sensitive supervised random walk model. The following three baselines combine the language attention network (LAN) as a subject filter with general influencer detection models: (4) LAN+ReF [13], a statistical and analytical model based on influence topology; (5) LAN+RR-LT [17], a polling-based method with a sample of random reversely reachable sets to approximate user influence; (6) LAN+CoupledGNN [3], two coupled graph neural networks to iteratively model and predict user influence.

Evaluation. We evaluate our DID approach against the baselines with four commonly used metrics: (1) F1; (2) Area Under Curve (AUC); (3) Normalized Discounted Cumulative Gain (NDCG); (4) Mean Average Precision (MAP).

Implementation Details. The language attention network has bidirectional GRUs with hidden dimensions in $\{50,100,200\}$ and the word embeddings are initialized with the Glove vectors [10] pre-trained on the Twitter corpus. We initialize the pool with 2 seed users and the final size of seed user pool varies in $[2,8]$. The influence convolution network is a two-layer network.

\subsection{Experimental Results}

Table 2 summarizes the model performance of detecting top- $t$ topicspecific influencers, where $t$ is the number of influencers in ground truth ( $t=20$ for HIV and $t=15$ for SuICIDE). Overall, DID is able to outperform other baselines across the two datasets on all metrics. We make the following observations. (1) Compared with TAP [12], TwitterRank [15] and TS-SRW that directly identify influencers in rare topics from the massive social messages, DID outperforms by iteratively learning an accurate subject filter. (2) As combination models, Filter+ReF [13], Filter+RR-LT [17] and Filter+CoupledGNN [3] train the influencer detection model with fixed social message filters. In contrast, our proposed DID can adaptively update the subject filter for better influencer detection performance. (3) We conduct the ablation study of DID by training a non-iterative LAN, removing interaction integration, and influence convolution one by one at a time. As the results on the HIV dataset shown in Table 3, each module contributes to the performance improvement, and the proposed DID benefits from iterative LAN, interaction integration and influence convolution. (4) We also investigate how seed 
Table 2: Topic-specific influencer detection performance.

\begin{tabular}{|c|c|c|c|c|c|c|c|c|}
\hline \multirow{2}{*}{ Methods } & \multicolumn{4}{|c|}{ HIV } & \multicolumn{4}{c|}{ SUICIDE } \\
\cline { 2 - 9 } & F1 & AUC & NDCG & MAP & F1 & AUC & NDCG & MAP \\
\hline TAP & 0.415 & 0.687 & 0.341 & 0.612 & 0.535 & 0.734 & 0.423 & 0.732 \\
TwitterRank & 0.212 & 0.585 & 0.081 & 0.578 & 0.374 & 0.659 & 0.186 & 0.742 \\
TS-SRW & 0.159 & 0.750 & 0.062 & 0.500 & 0.275 & 0.824 & 0.106 & 0.611 \\
\hline LAN-ReF & 0.602 & 0.819 & 0.415 & 0.695 & 0.434 & 0.727 & 0.231 & 0.751 \\
LAN+RR-LT & 0.683 & 0.793 & 0.500 & 0.877 & 0.731 & 0.863 & 0.558 & 0.930 \\
LAN+CoupledGNN & 0.719 & 0.870 & 0.529 & 0.910 & 0.642 & 0.829 & 0.683 & 0.956 \\
\hline DID & $\mathbf{0 . 8 8 2}$ & $\mathbf{0 . 9 1 2}$ & $\mathbf{0 . 7 7 1}$ & $\mathbf{0 . 9 7 0}$ & $\mathbf{0 . 8 5 9}$ & $\mathbf{0 . 9 3 6}$ & $\mathbf{0 . 7 3 9}$ & $\mathbf{0 . 9 9 6}$ \\
\hline
\end{tabular}

\begin{tabular}{|c|c|c|c|c|}
\hline & F1 & AUC & NDCG & MAP \\
\hline DID & $\mathbf{0 . 8 8 2}$ & $\mathbf{0 . 9 1 2}$ & $\mathbf{0 . 7 7 1}$ & $\mathbf{0 . 9 7 0}$ \\
\hline Non-iterative LAN & 0.791 & 0.852 & 0.730 & 0.933 \\
w/o interaction integration & 0.823 & 0.862 & 0.749 & 0.942 \\
w/o influence convolution & 0.801 & 0.878 & 0.753 & 0.961 \\
\hline
\end{tabular}

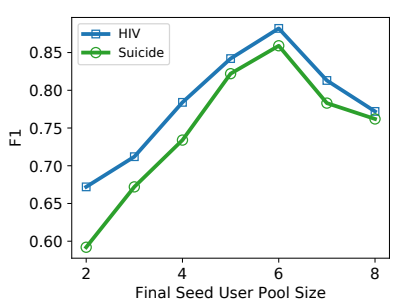

(a) User Pool Size

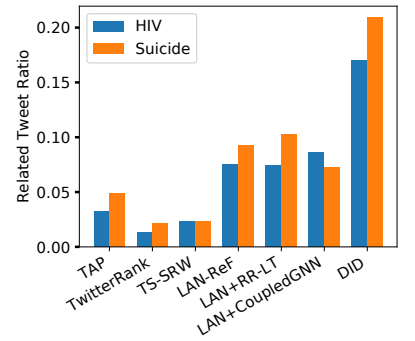

(b) Specificity
Figure 3: (a) F1 vs. seed user pool final size. (b) The specificity evaluation.

user pool size impacts the detection accuracy in DID, shown in Figure 3(a). Over the two datasets, we observe a common phenomenon: there exists an optimal size that delivers the best F1 score.

To confirm that the identified users indeed focus on the target rare topics, we conduct an experiment on their followers' tweets. With the output influencer set of each model, we randomly select 100 followers for each influencer and crawl follower's most recent 100 tweets. Figure 3(b) shows the percentage of subject-related tweets to all tweets. The proposed DID model has the highest ratio of related tweets in the two datasets, which indicates these influencers can deliver specific influence to their followers.

Table 4: Influencers detected in US-ENGLISH dataset.

\begin{tabular}{|l|l|}
\hline Topic & Output of DID model \\
\hline \hline \multirow{3}{*}{ HIV } & $\begin{array}{l}\text { CDC_HIVAIDS, talkHIV, PEPFAR, iasociety, } \\
\text { blackaids, WHO, TheBodyDotCom, } \\
\text { HIV_Insight, GlobalFund, GreaterThanAIDS }\end{array}$ \\
\hline \multirow{3}{*}{ Suicide } & $\begin{array}{l}\text { MentallyAwareNG,afspnational,samaritans,80- } \\
\text { 0273talk,Spreading_LOve,cspyyc,papyrus_tweets } \\
\text { CarlDunnJr, depressionnote, CharitySANE }\end{array}$ \\
\hline
\end{tabular}

To evaluate the scalability of the proposed DID, we show the top 10 topic-specific influencers on large scale US-ENGLISH dataset in Table 4. Account names in bold are topic-specific influencers in HIV or Suicide topic. The results show that DID is capable of detecting these topic-specific influencers out of 3.8 million users in the US-ENGLISH dataset.

\section{CONCLUSION}

This paper investigates the problem of detecting on-demand topicspecific influencers. We introduce a computational framework, onDemand Influencer Discovery model, based on the language attention network and influence convolution network. Comprehensive evaluations are conducted with three Twitter datasets and the results show promising performance of the proposed model against comparative baselines.

\section{ACKNOWLEDGEMENT}

We thank the anonymous reviewers for their careful reading and insightful comments on our manuscript. The work was partially supported by NIAID (R56AI125105, 7R01AI132030), NIMH (5R01MH106415), and NSF (DGE1829071, IIS-2031187).

\section{REFERENCES}

[1] Nicola Barbieri, Francesco Bonchi, and Giuseppe Manco. 2012. Topic-Aware Social Influence Propagation Models. In ICDM.

[2] Bin Bi, Yuanyuan Tian, Yannis Sismanis, Andrey Balmin, and Junghoo Cho. 2014. Scalable topic-specific influence analysis on microblogs. In WSDM.

[3] Qi Cao, Huawei Shen, Jinhua Gao, Bingzheng Wei, and Xueqi Cheng. 2020. Popularity Prediction on Social Platforms with Coupled Graph Neural Networks. In WSDM.

[4] Meeyoung Cha, Hamed Haddadi, Fabrício Benevenuto, and P. Krishna Gummadi. 2010. Measuring User Influence in Twitter: The Million Follower Fallacy. In ICWSM.

[5] Kiran Garimella, Ingmar Weber, and Munmun De Choudhury. 2016. Quote rts on twitter: usage of the new feature for political discourse. In WebSci.

[6] Jyun-Yu Jiang, Xue Sun, Wei Wang, and Sean Young. 2019. Enhancing Air Quality Prediction with Social Media and Natural Language Processing. In ACL.

[7] Georgios Katsimpras, Dimitrios Vogiatzis, and Georgios Paliouras. 2015. Determining influential users with supervised random walks. In $W W W$.

[8] David Kempe, Jon Kleinberg, and Éva Tardos. 2003. Maximizing the spread of influence through a social network. In $K D D$.

[9] Thomas N Kipf and Max Welling. 2017. Semi-supervised classification with graph

convolutional networks. In $I C L R$. Global Vectors for Word Representation. In EMNLP.

[11] Jiezhong Qiu, Jian Tang, Hao Ma, Yuxiao Dong, Kuansan Wang, and Jie Tang. 2018. DeepInf: Social Influence Prediction with Deep Learning. In KDD.

[12] Jie Tang, Jimeng Sun, Chi Wang, and Zi Yang. 2009. Social influence analysis in large-scale networks. In $K D D$.

[13] Ramine Tinati, Leslie Carr, Wendy Hall, and Jonny Bentwood. 2012. Identifying communicator roles in twitter. In $W W W$

[14] Wei Wei, Gao Cong, Chunyan Miao, Feida Zhu, and Guohui Li. 2016. Learning to find topic experts in twitter via different relations. TKDE (2016).

[15] Jianshu Weng, Ee-Peng Lim, Jing Jiang, and Qi He. 2010. Twitterrank: finding topic-sensitive influential twitterers. In WSDM.

[16] Keyulu Xu, Weihua Hu, Jure Leskovec, and Stefanie Jegelka. 2018. How powerful are graph neural networks? arXiv (2018).

[17] Yu Yang, Zhefeng Wang, Jian Pei, and Enhong Chen. 2017. Tracking Influential Individuals in Dynamic Networks. TKDE (2017).

[18] Wenchao Yu, Cheng Zheng, Wei Cheng, Charu C Aggarwal, Dongjin Song, Bo Zong, Haifeng Chen, and Wei Wang. 2018. Learning deep network representations with adversarially regularized autoencoders. In KDD.

[19] Cheng Zheng, Jyun-Yu Jiang, Yichao Zhou, Sean D Young, and Wei Wang. 2020. Social Media User Geolocation via Hybrid Attention. In SIGIR.

[20] Cheng Zheng, Qin Zhang, Guodong Long, Chengqi Zhang, Sean D Young, and Wei Wang. 2020. Measuring Time-Sensitive and Topic-Specific Influence in Social Networks With LSTM and Self-Attention. IEEE Access (2020) 